\title{
Trends in outpatient antibiotic use and prescribing practice among US older adults, 2011-15: observational study
}

\author{
Scott W Olesen, ${ }^{1}$ Michael L Barnett, ${ }^{2,3}$ Derek R MacFadden, ${ }^{4,5}$ Marc Lipsitch, ${ }^{1,5}$ \\ Yonatan $\mathrm{H} \mathrm{Grad}^{1,6}$
}

${ }^{1}$ Department of Immunology

and Infectious Diseases,

Harvard T H Chan School of

Public Health, Boston, MA, USA

${ }^{2}$ Department of Health Policy and Management, Harvard TH

Chan School of Public Health, Boston, MA, USA

${ }^{3}$ Division of General Internal Medicine and Primary Care,

Department of Medicine,

Brigham and Women's Hospital, Harvard Medical School,

Boston, MA, USA

${ }^{4}$ Division of Infectious Diseases, Department of Medicine,

University of Toronto, Toronto,

ON, Canada

${ }^{5}$ Center for Communicable

Disease Dynamics, Department

of Epidemiology, Harvard TH

Chan School of Public Health,

Boston, MA, USA

${ }^{6}$ Division of Infectious Diseases, Brigham and Women's Hospital, Harvard Medical School,

Boston, MA, USA

Correspondence to:

H Grad ygrad@hsph.harvard.edu (or @yhgrad on Twitter;

ORCID 0000-0001-5646-1314) Additional material is published online only. To view please visit the journal online.

Cite this as: $B M J$ 2018;362:k3155 http://dx.doi.org/10.1136/bmi.k3155

Accepted: 4 July 2018

\section{ABSTRACT}

OBJECTIVE

To identify temporal trends in outpatient antibiotic use and antibiotic prescribing practice among older adults in a high income country.

\section{DESIGN}

Observational study using United States Medicare administrative claims in 2011-15.

SETTING

Medicare, a US national healthcare program for which $98 \%$ of older adults are eligible.

\section{PARTICIPANTS}

4.5 million fee-for-service Medicare beneficiaries aged 65 years old and older.

\section{MAIN OUTCOME MEASURES}

Overall rates of antibiotic prescription claims, rates of potentially appropriate and inappropriate prescribing, rates for each of the most frequently prescribed antibiotics, and rates of antibiotic claims associated with specific diagnoses. Trends in antibiotic use were estimated by multivariable regression adjusting for beneficiaries' demographic and clinical covariates.

RESULTS

The number of antibiotic claims fell from 1364.7 to 1309.3 claims per 1000 beneficiaries per year in 2011-14 (adjusted reduction of 2.1\% (95\% confidence interval $2.0 \%$ to $2.2 \%$ )), but then rose to 1364.3 claims per 1000 beneficiaries per year in 2015 (adjusted reduction of $0.20 \%$ over 2011 $15(0.09 \%$ to $0.30 \%)$ ). Potentially inappropriate antibiotic claims fell from 552.7 to 522.1 per 1000 beneficiaries over 2011-14, an adjusted reduction of $3.9 \%$ (3.7\% to $4.1 \%)$. Individual antibiotics had heterogeneous changes in use. For example, azithromycin claims per beneficiary decreased by $18.5 \%(18.2 \%$ to $18.8 \%)$ while levofloxacin claims increased by $27.7 \%$ (27.2\% to $28.3 \%$ ). Azithromycin

\section{WHAT IS ALREADY KNOWN ON THIS TOPIC}

Inappropriate antibiotic use is widespread, and antibiotic use is higher among older adults

Antibiotic use, which has been mostly steady in high income countries, could have fallen in the United States in recent years

\section{WHAT THIS STUDY ADDS}

From 2011 to 2015, overall antibiotic use remained relatively steady, but the use of individual antibiotics underwent large and variable changes

Potentially inappropriate use of antibiotics decreased slightly in 2011-14, but prescribers' use of individual antibiotics had no consistent association with guideline changes, and were perhaps instead driven by market factors and concerns about safety use associated with each of the potentially appropriate and inappropriate respiratory diagnoses decreased, while levofloxacin use associated with each of those diagnoses increased.

\section{CONCLUSIONS}

Among US Medicare beneficiaries, overall antibiotic use and potentially inappropriate use in 2011-15 remained steady or fell modestly, but individual drugs had divergent changes in use. Trends in drug use across indications were stronger than trends in use for individual indications, suggesting that guidelines and concerns about antibiotic resistance were not major drivers of change in antibiotic use.

\section{Introduction}

High rates of antibiotic prescribing are a major challenge to public health. ${ }^{12}$ Clinically inappropriate antibiotic prescribing comprises a large fraction of overall use and contributes to increasingly broad antibiotic resistance. ${ }^{3-6}$ Despite guidelines and calls by government agencies, professional medical societies, and other organizations to reduce antibiotic prescribing for inappropriate indications, ${ }^{78}$ prescribing patterns have changed little, ${ }^{9-11}$ and antibiotic use in high income countries remains steady. ${ }^{12}$ Furthermore, data on antibiotic prescribing trends that can guide stewardship efforts remain sparse, particularly for key vulnerable populations, such as older adults.

Older adults are a particularly important population with respect to antibiotic overuse. They use about 50\% more antibiotics per capita than younger adults ${ }^{13-15}$ and have the highest risk of poor outcomes from the adverse effects of antibiotics, including Clostridium difficile infection. ${ }^{16}$ In the United States, after several decades of steady antibiotic use, ${ }^{17-19}$ during which use of individual drugs has varied, ${ }^{18-21}$ overall antibiotic use may have begun to fall slightly. ${ }^{72-25}$ However, recent trends in antibiotic use among older adults are unclear, perhaps having hit a peak in 2006 and a trough in $2014 .{ }^{25}{ }^{26}$ Furthermore, there are limited data on the trends for individual antibiotics and on the use of individual antibiotics in association with specific indications. More definitive evidence on antibiotic use and its appropriateness in older adults is needed to guide stewardship interventions in this critical population.

Therefore, we investigated recent trends in antibiotic prescribing among older adults, using administrative claims from the Medicare program from 2011 to 2015. About $98 \%$ of Americans over 65 years old are eligible for Medicare, a national program administered by the Centers for Medicare and Medicaid Services (CMS) that includes hospital health insurance (Medicare 
Part A), outpatient healthcare insurance (Part B), and prescription drug plans (Part D). ${ }^{27}$ We focused on trends in potentially inappropriate antibiotic use and heterogeneity in trends among individual antibiotics to identify potential targets for future stewardship interventions.

\section{Methods}

\section{Study population}

We studied a random $20 \%$ sample of beneficiaries enrolled in Medicare medical insurance (Parts A and B) and prescription drug coverage (Part D) for 2011 to 2015. The $20 \%$ sample is determined by Medicare using beneficiaries' identification numbers. ${ }^{27}$ For each data year, we included only individuals who were continuously enrolled in fee-for-service Medicare Parts A, B, and D for the entire year and who were at least 65 years old. Beneficiaries who opted to join managed care plans (Medicare Advantage) rather than traditional fee-for-service plans were excluded, because CMS only records Part D claims for beneficiaries on fee-forservice plans.

\section{Demographic and clinical variables}

To characterize the differences in antibiotic use among older adults, we captured beneficiary sex (male or female), race/ethnicity (white, black, Hispanic, other), age (stratified into 65-74, 75-84, 85-94, and $\geq 95$ year old groups), US Census region (Northeast, South, Midwest, West), eligibility for Medicaid (a US program that provides health insurance to low income people), and the presence of 20 chronic conditions. Of the 27 chronic conditions defined by CMS in its Chronic Condition Warehouse database, we selected the 20 conditions with one-year reference periods to prevent a bias toward more chronic conditions among individuals who have been Medicare beneficiaries for more years.

\section{Classifying antibiotic claims}

We examined original (that is, not refill) outpatient pharmacy claims (Medicare Part D Events file) for oral and injected prescription antibiotics (appendix table 1), as defined using the Medicare Formulary file and aggregated by generic antibiotic formulation (appendix table 2). We excluded refills because original pharmacy claims approximate prescriptions, a typical measure of antibiotic use in US studies, ${ }^{213}$ and because we did not expect refills to have associated prescriber encounters that would allow for assessment of prescribing practice. We also treated multiple claims from the same beneficiary on the same day for the same generic antibiotic as one claim, which excluded less than $1 \%$ of claims. To determine how individual antibiotics contributed to overall use and trends, we examined both overall antibiotic claims and claims for each of the 10 most frequently prescribed antibiotics. Medicare claims data do not include information about antibiotics dispensed in inpatient facilities such as hospitals or skilled nursing facilities.

\section{Encounters and diagnoses}

We linked antibiotic claims with outpatient prescriber encounters (Medicare Part B, Carrier and Outpatient files) and inpatient encounters (Part A, Inpatient and Skilled Nursing Facility files). An antibiotic claim was linked with an outpatient encounter if the prescription claim occurred on the day of the encounter or up to seven days after. A claim was linked with an inpatient encounter if the claim was on the discharge date or up to seven days after.

ICD-9 (international classification of diseases, 9th revision) diagnosis codes recorded for these encounters were grouped into 20 diagnostic categories (eg, pneumonia) by use of a previously published classification scheme from a US Centers for Disease Control and Prevention workgroup, described by Fleming-Dutra and colleagues. ${ }^{2}$ We did not follow the exception listed in the "Bronchitis, bronchiolitis" category, "Excludes visits in which the 2nd or 3rd diagnosis was chronic bronchitis, emphysema, or COPD," because this exception was specific to the coding format in their data source. ${ }^{28}$ We also followed that study's organization of diagnostic categories into tiers of antibiotic appropriateness: tier 1 (antibiotics almost always indicated), tier 2 (antibiotics may be indicated), and tier 3 (antibiotics not indicated).

We allowed multiple diagnoses to be linked to each claim and classified antibiotic claims with an associated tier 1 or tier 2 diagnosis as potentially appropriate. We classified claims with only tier 3 diagnoses as potentially inappropriate. We did not classify antibiotics without associated diagnoses as appropriate or inappropriate. Linking multiple diagnoses to a single claim is conservative because only one code for an antibiotic appropriate diagnosis is needed to classify the claim as potentially appropriate. This definition of appropriateness differs from that used by Fleming-Dutra and colleagues. ${ }^{2}$ In that study, tier 1 diagnoses were appropriate, most tier 3 diagnoses were inappropriate, and appropriate antibiotic prescribing rates for the remaining diagnoses were determined by surveys of pathogen carriage or the minimum prescribing rate across the four US Census regions. We used a different definition of inappropriate use because Fleming-Dutra and colleagues' methodology was not designed for trend studies. Pathogen carriage and geographical differences in prescribing rates themselves change with time-thus, for example, if antibiotic use in the lowest use region declined, the inappropriate prescribing rate in other regions would appear to rise.

In October 2015, the US healthcare system transitioned from ICD-9 to ICD-10 diagnosis codes, but the scheme in the Fleming-Dutra study ${ }^{2}$ used ICD-9 codes. To avoid bias from coding changes, we did not link antibiotic claims in 2015 with diagnoses. Therefore, we restricted all analyses involving diagnoses to years 2011-14.

\section{Outcomes}

To assess trends in antibiotic use, the outcome of interest was the number of antibiotic claims per 
1000 beneficiaries in each year. We considered total antibiotic claims, potentially appropriate claims, potentially inappropriate claims, claims without associated diagnoses, and claims for each of the 10 frequently prescribed antibiotics. Rates of potentially appropriate claims, potentially inappropriate claims, and claims without associated diagnoses were not computed for 2015.

To assess how changes in prescribing practice contributed to the trends in use of individual drugs, the outcome of interest was the number of claims for a particular drug linked with a particular diagnosis (eg, number of azithromycin claims associated with a pneumonia diagnosis) per 1000 beneficiaries in a year. We considered the 13 diagnosis categories with the most associated antibiotic claims, grouped those diagnosis categories into four infection sites (respiratory, gastrointestinal, genitourinary, and skin/cutaneous/ mucosal), and considered the three antibiotics contributing the most claims for each infection site. In analyses of each infection site, we allowed one claim to be linked to multiple diagnoses. In a sensitivity analysis designed to evaluate whether this multiple linking introduced a bias into the measured trends in prescribing practice, a claim was linked with only one diagnosis per infection site. If multiple links were possible, a single diagnosis was selected at random using PROC SURVEYSELECT in SAS (version 9.4).

\section{Statistical analyses}

To assess trends in the study population characteristics, we performed Poisson regressions (for number of beneficiaries and mean number of chronic conditions) or log-binomial regressions (for proportion of female, white, dual eligible participants for Medicaid, in each age group, and in each Census region) predicting the population characteristic from study year. Adjusted trends in antibiotic use (that is, claims per beneficiary per year) were assessed by Poisson regression. The main covariate of interest was a linear term for year. We adjusted for beneficiary age group, sex, race, Census region, dual eligibility, and number of chronic conditions. When reporting a trend in use, the coefficient for year was projected to the full 2011-15 time span for all regressions. Trends in prescribing practice were projected to the 2011-14 time span. Regressions were performed by PROC GENMOD. Clustered standard error estimators accounting for correlations between multiple measurements from the same beneficiaries ${ }^{29}$ yielded similar confidence intervals. We reported 95\% confidence intervals.

\section{Patient involvement}

No patients were involved in setting the research question or the outcome measures, nor were they involved in developing plans for design or implementation of the study. No patients were asked to aid in interpreting or disseminating the results. There are no plans to disseminate the results of the research to the relevant patient community.

\section{Results}

Our study sample included 4.5 million unique Medicare beneficiaries with 19.5 million antibiotic claims from 2011 to 2015 (table 1). Population characteristics changed by less than $10 \%$ over the study period except for the number of beneficiaries (appendix table 3) and proportion of beneficiaries eligible for Medicaid (appendix table 4).

Use of all antibiotics fell from 1364.7 to 1309.3 claims per 1000 beneficiaries during 2011-14 (adjusted 2.1\% decrease over 2011-14 period, 95\% confidence interval $2.0 \%$ to $2.2 \%$; fig $1 \mathrm{~A}$ ), but then increased to 1364.3 in 2015 (adjusted $0.20 \%$ decrease over $2011-15$ period, $0.09 \%$ to $0.30 \%$; fig $1 \mathrm{~A}$, fig 2). Trends in use varied by age group, race, and region, and use may have increased among beneficiaries aged 75 to 84 years, female beneficiaries, white beneficiaries, and beneficiaries in the South and Northeast Census regions (appendix table 5).

Potentially inappropriate prescribing changed from 552.7 to 552.1 claims per 1000 beneficiaries during 2011-14 (fig 1A), an adjusted decrease of 3.9\% (95\% confidence interval $3.7 \%$ to $4.1 \%$ ), while potentially

\begin{tabular}{|c|c|c|c|c|c|}
\hline & \multicolumn{5}{|l|}{ Year } \\
\hline & 2011 & 2012 & 2013 & 2014 & 2015 \\
\hline \multicolumn{6}{|l|}{ Age (years; \%) } \\
\hline $65-74$ & 47.5 & 48.3 & 49.2 & 50.3 & 49.6 \\
\hline $75-84$ & 35.1 & 34.5 & 34.1 & 33.6 & 33.3 \\
\hline$\geq 95$ & 1.67 & 1.64 & 1.55 & 1.50 & 1.73 \\
\hline No of chronic conditions (mean (SD)) & $2.75(1.80)$ & $2.74(1.81)$ & $2.73(1.80)$ & $2.71(1.80)$ & $2.76(1.86)$ \\
\hline Female (\%) & 63.0 & 62.3 & 61.1 & 61.2 & 62.5 \\
\hline White (\%) & 81.4 & 81.5 & 82.2 & 82.8 & 83.4 \\
\hline Medicaid eligible (\%) & 25.0 & 23.6 & 20.1 & 18.3 & 16.8 \\
\hline North east & 18.5 & 19.4 & 19.7 & 19.9 & 20.3 \\
\hline
\end{tabular}




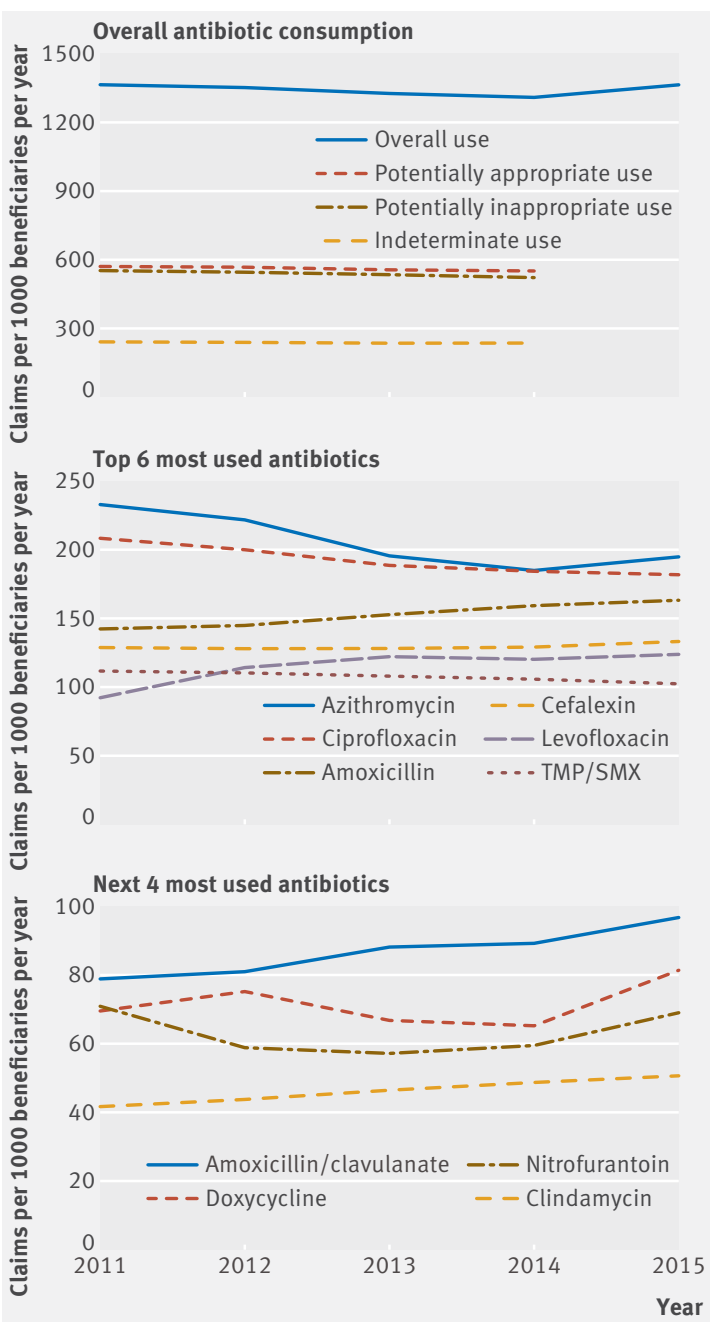

Fig 1 | Rates of antibiotic prescribing among Medicare beneficiaries aged 65 years and older, 2011-15. Lines indicate claims per 1000 beneficiaries per year for (A) all antibiotic claims, potentially appropriate claims, potentially inappropriate claims, and claims without associated diagnoses (indeterminate use); and for claims for the (B) six most claimed antibiotics and (C) four next most claimed antibiotics. Potentially appropriate, potentially appropriate, and indeterminate use were not calculated for 2015. TMP/SMX=trimethoprim/ sulfamethoxazole

appropriate prescribing remained nearly steady, changing from 571 to 551 claims per 1000 beneficiaries during 2011-14 (adjusted $0.2 \%$ decrease, $0.4 \%$ to $0.1 \%$ ). The proportion of claims that were potentially inappropriate remained nearly steady, changing from $40.5 \%$ in 2011 to $39.9 \%$ in 2014 .

The 10 most frequently prescribed antibiotics, which accounted for $87 \%$ of all antibiotic claims, showed heterogeneous trends (fig 1B, fig 1C, fig 2). Use fell for azithromycin, ciprofloxacin, and trimethoprim/ sulfamethoxazole, but use rose for the seven other drugs. The most marked changes were in azithromycin (adjusted decline of $18.5 \%, 95 \%$ confidence interval $18.2 \%$ to $18.8 \%$ ) and levofloxacin (adjusted increase of $27.7 \%, 27.2 \%$ to $28.3 \%$ ).
For levofloxacin, amoxicillin/clavulanate, and azithromycin, trends in use were similar across antibiotic appropriate and antibiotic inappropriate respiratory condition diagnoses. Use of levofloxacin, which increased overall, also increased in association with each of the respiratory diagnostic categories we considered (both antibiotic appropriate and inappropriate): pneumonia, sinusitis, viral upper respiratory tract infections, bronchitis, asthma and allergy, and other respiratory conditions (table 2). Use of amoxicillin/clavulanate, which increased overall, also increased in association with each of these respiratory diagnoses except for pneumonia. By contrast, use of azithromycin, which decreased overall, decreased in association with each of these appropriate and inappropriate diagnostic categories.

For example, use of azithromycin following pneumonia diagnoses, for which antibiotics are considered appropriate, fell from 17.3 claims per 1000 beneficiaries in 2011 to 13.9 claims per 1000 beneficiaries in 2014 (adjusted 17.0\% decrease (95\% confidence interval $15.9 \%$ to $18.0 \%$ ); table 2). Use of azithromycin following a diagnosis for viral upper respiratory tract infection, for which antibiotics are considered inappropriate, fell from 60.0 to 52.3 claims per 1000 beneficiaries per year in 2011-14 (adjusted $12.2 \%$ decrease $(8.5 \%$ to $9.7 \%))$. By contrast, use of levofloxacin for pneumonia rose from 28.4 to 35.0 claims per 1000 beneficiaries in 2011-14 (adjusted $27.1 \%$ increase $(26.0 \%$ to $28.2 \%)$ ), and use of levofloxacin for viral upper respiratory tract infection rose from 19.2 to 29.2 claims per 1000 beneficiaries in 2011-14 (adjusted 53.1\% increase (51.6\% to 54.7\%)). When linking each claim with only one diagnosis, the number of claims associated with each diagnosis was lower, but the trends in prescribing practice were nearly identical (appendix table 6).

Prescribing practice patterns for gastrointestinal infections, for which antibiotics are potentially appropriate, and other gastrointestinal conditions, for which antibiotics are inappropriate, displayed a similar pattern of use across antibiotic appropriate and inappropriate diagnoses (appendix table 7). Use of ciprofloxacin, which decreased overall, also fell in association with both infections and other conditions, while use of levofloxacin, which rose overall, also rose in association with both infections and other conditions. Use of metronidazole, which decreased overall (adjusted reduction of 5.0\% (95\% confidence interval $4.3 \%$ to $5.8 \%$ )), fell in association with infections but not with other gastrointestinal conditions. Prescribing practice for genitourinary and skin/cutaneous/ mucosal conditions also largely showed the pattern of increases or decreases by antibiotic across indications, regardless of appropriateness of the diagnosis (appendix tables 8 and 9).

\section{Discussion}

\section{Principal findings}

In this analysis of a national sample of Medicare beneficiaries, we found that overall antibiotic use 


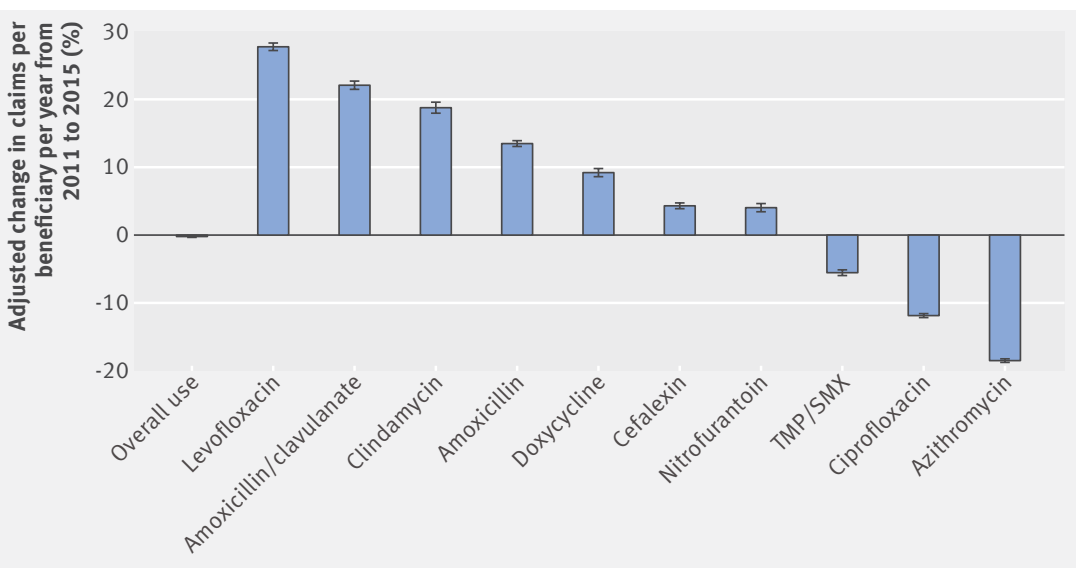

Fig 2 | Adjusted trends in antibiotic use among Medicare beneficiaries aged 65 years and older, 2011-15. Bars indicate relative changes in claims per beneficiary per year for all antibiotic claims and for the 10 most claimed antibiotics. Relative changes were determined by Poisson regression on claims per beneficiary per year, adjusted for age, sex, race, census region, dual eligibility, and number of chronic conditions. Error bars $=95 \%$ confidence intervals; TMP $/ S M X=$ trimethoprim/sulfamethoxazole

remained nearly constant during 2011-15, and potentially inappropriate prescribing declined 4\% during 2011-14. We saw an increased use of levofloxacin over azithromycin for all respiratory conditions during this period, irrespective of whether antibiotics were appropriate for that indication or not. Prescribing practice for other conditions displayed similar patterns, suggesting that changes in antibiotic prescribing practice among US older adults could be due to shifting preferences for antibiotics across indications rather than targeted reductions in use of particular antibiotics for particular indications. Furthermore, the proportion of antibiotic use we observed as potentially inappropriate (about 40\%; fig 1A) was substantially higher than previously reported ${ }^{2}(18 \%$ for people aged $\geq 65$ years old), indicating that inappropriate antibiotic prescribing among Medicare beneficiaries might be much higher than expected.

There are at least four explanations for the trends we observed in the use of individual antibiotics. Our results are inconsistent with the first two explanations but consistent with the second two. Firstly, trends could represent altered prescribing practice in response to changed prescribing guidelines. For example, in 2007, the Infectious Diseases Society of America and the American Thoracic Society issued a recommendation that a respiratory fluoroquinolone be used instead of a macrolide for treating community acquired pneumonia in certain patients. $^{30}$ If this recommendation were still in the process of being implemented in 201114 , it could help explain why azithromycin use for pneumonia decreased by $17 \%$ while use of levofloxacin for pneumonia increased by $27 \%$ (table 2). Secondly, the trends could reflect concerns about antibiotic resistance, such as rising macrolide resistance among community-acquired pneumonia, ${ }^{31}$ which could also explain decreased use of azithromycin for pneumonia. In this study, we found that use of azithromycin for all respiratory conditions fell, while use of levofloxacin for those conditions rose. This pattern is inconsistent with diagnosis-specific explanations.

We formulated two alternative explanations that are consistent with trends in antibiotic use across diagnoses. Firstly, the trends could reflect safety concerns about particular antibiotics. For example, in 2013, the US Food and Drug Administration warned prescribers that azithromycin might increase the risk of cardiovascular death, ${ }^{32}$ which could have contributed to the reduced popularity of azithromycin across diagnoses. Secondly, these trends might represent market factors such as pricing, availability, and advertising, as suggested in a previous study, ${ }^{19}$ which found that broad spectrum antibiotics were used more for both antibiotic appropriate and inappropriate indications for respiratory disorders in 1995-2002. Thus, our results suggest that the observed trends in drug use are driven more by safety concerns or market factors than by altered guidelines or by concerns about antibiotic resistance. Furthermore, the slow decline in overall antibiotic use we observed, coupled with the fast trends in use of individual drugs, suggests that it is easier to substitute one antibiotic for another than

\begin{tabular}{|c|c|c|c|c|c|c|c|c|c|c|}
\hline \multirow[b]{3}{*}{ Diagnosis§ } & \multirow[b]{3}{*}{$\begin{array}{l}\text { Antibiotic appro- } \\
\text { priate diagnosis?† }\end{array}$} & \multicolumn{9}{|c|}{ Antibiotic use (No of claims per 1000 beneficiaries per year ${ }^{\star}$ ) } \\
\hline & & \multicolumn{3}{|c|}{ Azithromycin } & \multicolumn{3}{|c|}{ Levofloxacin } & \multicolumn{3}{|c|}{ Amoxicillin/clavulanate } \\
\hline & & 2011 & 2014 & $\begin{array}{l}\text { Relative change } \\
(\% ; 95 \% \mathrm{Cl}) \neq\end{array}$ & 2011 & 2014 & $\begin{array}{l}\text { Relative change } \\
(\% ; 95 \% \mathrm{Cl}) \neq\end{array}$ & 2011 & 2014 & $\begin{array}{l}\text { Relative change } \\
(\% ; 95 \% \mathrm{Cl}) \neq\end{array}$ \\
\hline Pneumonia & Yes & 17.3 & 13.9 & $-17.0(-18.0$ to -15.9$)$ & 28.4 & 35.0 & 27.1 (26.0 to 28.2$)$ & 8.6 & 8.3 & $3.0(1.3$ to 4.8$)$ \\
\hline Sinusitis & Potentially & 29.9 & 24.6 & $-21.2(-21.9$ to -20.4$)$ & 6.9 & 12.5 & $64.4(61.8$ to 67.0$)$ & 15.4 & 21.0 & $35.2(33.6$ to 36.8$)$ \\
\hline $\begin{array}{l}\text { Viral upper respiratory } \\
\text { tract infections }\end{array}$ & No & 60.0 & 52.3 & $-12.2(-12.8$ to -11.6$)$ & 19.2 & 29.2 & 53.1 (51.6 to 54.7$)$ & 10.8 & 14.0 & 31.9 (30.0 to 33.7) \\
\hline Acute bronchitis & No & 55.9 & 43.8 & $-20.9(-21.5$ to -20.4$)$ & 16.1 & 23.8 & $44.9(44.3$ to 46.4$)$ & 8.6 & 9.5 & $13.3(11.5$ to 15.2$)$ \\
\hline $\begin{array}{l}\text { Other respiratory dis- } \\
\text { orders }\end{array}$ & No & 60.9 & 49.8 & $-15.4(-16.0$ to -14.8$)$ & 55.3 & 69.1 & 29.9 (29.0 to 30.8) & 23.3 & 24.3 & $9.2(8.1$ to 10.4$)$ \\
\hline Asthma and allergy & No & 21.4 & 18.6 & $-13.0(-14.0$ to -12.0$)$ & 8.3 & 12.1 & $45.2(43.0$ to 47.5$)$ & 5.8 & 7.4 & 28.2 (25.7 to 30.8$)$ \\
\hline \multicolumn{11}{|c|}{$\begin{array}{l}\text { *Includes claims for each antibiotic linked to the given diagnosis. A single claim might be linked to multiple diagnoses, so the sum of the } 2011 \text { and } 2014 \text { columns exceeds the number of claims } \\
\text { associated with any listed diagnosis (see methods, and appendix table 6). } \\
\text { †Appropriateness of antibiotics for that diagnosis as determined by the US Centers for Disease Control and Prevention working group }{ }^{2} \text { (see methods). } \\
\text { łAdjusted value for } 2011-14 \text { change from Poisson regression on claims per beneficiary per year adjusted for age, sex, race, Census region, dual eligibility, and number of chronic conditions. } \\
\S \text { Pneumonia includes Streptococcus pneumoniae pneumonia, other bacterial pneumonia, pneumonia due to other specified organism, pneumonia in infectious diseases classified elsewhere, } \\
\text { bronchopneumonia with organism unspecified, and pneumonia with organism unspecified. Viral upper respiratory tract infections includes acute nasopharyngitis, acute laryngitis and tracheitis, } \\
\text { acute upper respiratory infections of multiple or unspecified sites, and cough. Acute bronchitis includes bronchitis not specified as acute or chronic, acute bronchitis, and bronchiolitis. Other } \\
\text { respiratory disorders include chronic bronchitis, dyspnea, stridor, hemoptysis, and abnormal sputum. Asthma and allergy includes allergic rhinitis and unspecified allergy. }\end{array}$} \\
\hline
\end{tabular}


to reduce overall antibiotic use, ${ }^{33}$ as happened in Finland as macrolide use decreased. ${ }^{34}$ We therefore speculate that the 2016 recommendations against the use of levofloxacin for uncomplicated respiratory conditions $^{35}$ might reverse the trend in levofloxacin use and cause a trade-off toward other drugs, such as azithromycin or amoxicillin/clavulanate, for example.

\section{Limitations}

Our study has several limitations. Firstly, the feefor-service beneficiaries-for which we have more complete prescription and encounter data-might not be representative of the entire US older adult population. ${ }^{36}$ Whether trends among US older adults are representative of trends among older adults in high income countries more generally is unclear. Secondly, the time period of our study prevented assessment of longer term trends. Thirdly, our study design could not determine whether changes in antibiotic use were due to changes in the behavior of individual prescribers or due to a changing composition of prescribers with different behaviors.

We also note caveats to interpreting the diagnosis antibiotic links and the classification of antibiotic use as potentially appropriate or inappropriate. Firstly, prescribing appropriateness is related to choice of antibiotic as well as many factors beyond those recorded in administrative claims data. Any classification of antibiotic use as appropriate or not must be considered approximate. Secondly, pharmacy claims data do not contain information about the condition the drug is intended to treat, such that we were not able to classify the $18 \%$ of antibiotic claims without associated diagnoses as appropriate or inappropriate. ${ }^{3738}$ Thirdly, trends in appropriateness could have been affected by trends in coding practice. ${ }^{39}{ }^{40}$ But we expect the most likely coding trend to be that truly inappropriate prescribing would be increasingly coded with antibiotic appropriate diagnoses, such that our observed decline in inappropriate use was an overestimate. Finally, the difference between the potentially inappropriate prescribing proportion in this study (40\%) and that in a previous report ${ }^{2}$ (18\% among Americans at least 65 years old) is likely due to the different data sources and methods of linking antibiotic claims with diagnoses in the two studies. In Fleming-Dutra and colleagues' data source, ${ }^{2}$ each antibiotic prescription was definitively associated with its motivating diagnosis, and the definitions of inappropriate use were differed to ours. These differences highlight the challenges in quantifying inappropriate antibiotic use and the need for consistent definitions of appropriate antibiotic use that can be applied across data sources. ${ }^{425}$

\section{Conclusions and policy implications}

We found that overall antibiotic use and potentially inappropriate antibiotic prescribing remained steady or decreased modestly in a national population of US older adults from 2011 to 2015. For drugs used to treat respiratory infections, most changes in antibiotic use were due to increased use of one antibiotic over another across indications, rather than clinically oriented changes in use. In our study, changes in antibiotic prescribing practice reflected shifting use between antibiotics rather than decreasing inappropriate prescribing across antibiotics, which implied that attempts to reduce outpatient antibiotic prescribing could generally result in a shifting palette, rather than a reduced amount, of antibiotic use. Because decades of effort to reduce outpatient antibiotic prescribing have improved the appropriateness of antibiotic use at most incrementally, we propose that stewardship programs instead focus on the apparently more feasible goal of shifting prescribing from newer, broader spectrum drugs to older, narrow spectrum drugs. The generally weak effects of outpatient stewardship programs ${ }^{41}$ and our results in particular suggest that strong policy changes and innovative approaches to stewardship will be necessary to substantially improve antibiotic prescribing practice.

Contributors: SWO, MLB, and YHG conceived of and designed the work. SWO analyzed the data. SWO and YHG drafted the work. All authors revised the work critically and approved the final manuscript. YHG is the guarantor. The corresponding author attests that all listed authors meet authorship criteria and that no others meeting the criteria have been omitted.

Funding: SWO and ML were supported by cooperative agreement U54GM088558 from the US National Institute of General Medical Sciences. The content is solely the responsibility of the authors and does not necessarily represent the official views of the National Institute of General Medical Sciences or the National Institutes of Health. This funding source had no role in the design of this study and will not have any role during its execution, analyses, interpretation of the data, or decision to submit results.

Competing interests: All authors have completed the ICMJE uniform disclosure form at www.icmje.org/coi_disclosure.pdf and declare: support from the US National Institute of General Medical Sciences fo the submitted work; no financial relationships with any organizations that might have an interest in the submitted work in the previous three years; no other relationships or activities that could appear to have influenced the submitted work.

Ethical approval: This study was deemed exempt from review by the institutional review board at the Harvard $\mathrm{T} H$ Chan School of Public Health, Boston, MA, USA.

Data sharing: All data are available from the Centers for Medicare and Medicaid Services.

Transparency: The lead author (the manuscript's guarantor) affirms that the manuscript is an honest, accurate, and transparent account of the study reported; that no important aspects of the study have been omitted; and that any discrepancies from the study as planned have been explained.

This is an Open Access article distributed in accordance with the Creative Commons Attribution Non Commercial (CC BY-NC 4.0) license, which permits others to distribute, remix, adapt, build upon this work non-commercially, and license their derivative works on different terms, provided the original work is properly cited and the use is noncommercial. See: http://creativecommons.org/licenses/by-nc/4.0/

1 Shapiro DJ, Hicks LA, Pavia AT, Hersh AL. Antibiotic prescribing for adults in ambulatory care in the USA, 2007-09. J Antimicrob Chemother 2014:69:234-40. doi:10.1093/jac/dkt301

2 Fleming-Dutra KE, Hersh AL, Shapiro DJ, et al. Prevalence of inappropriate antibiotic prescriptions among US ambulatory care visits, 2010-2011. JAMA 2016;315:1864-73. doi:10.1001/ jama.2016.4151

3 Gonzales R, Malone DC, Maselli JH, Sande MA. Excessive antibiotic use for acute respiratory infections in the United States. Clin Infect Dis 2001;33:757-62. doi:10.1086/322627

4 Smith DRM, Dolk FCK, Pouwels KB, Christie M, Robotham JV, Smieszek T. Defining the appropriateness and inappropriateness of antibiotic prescribing in primary care. / Antimicrob Chemother 2018;73(suppl_2):ii11-8. doi:10.1093/jac/dkx503 
5 Pouwels KB, Dolk FCK, Smith DRM, Robotham JV, Smieszek T. Actual versus 'ideal' antibiotic prescribing for common conditions in English primary care. J Antimicrob Chemother 2018;73(suppl_2):19-26. doi:10.1093/jac/dkx502

6 Almalki ZS, Alahmari AK, Guo JJ, Cavanaugh TM. Off-label use of oral fluoroquinolone antibiotics in outpatient settings in the United States, 2006 to 2012. Pharmacoepidemiol Drug Saf 2016;25:104251. doi: $10.1002 / p d s .4021$

7 Centers for Disease Control and Prevention. Antibiotic prescribing and use in the US. 2017 [cited 2018 Mar 19]. https://www.cdc.gov/ antibiotic-use/stewardship-report/index.html

8 Smieszek T, Pouwels KB, Dolk FCK, et al. Potential for reducing inappropriate antibiotic prescribing in English primary care. J Antimicrob Chemother 2018;73(suppl_2):ii36-43. doi:10.1093/jac/ dkx500

9 Barnett ML, Linder JA. Antibiotic prescribing to adults with sore throat in the United States, 1997-2010. JAMA Intern Med 2014;174:13840. doi:10.1001/jamainternmed.2013.11673

10 Barnett ML, Linder JA. Antibiotic prescribing for adults with acute bronchitis in the United States, 1996-2010. JAMA 2014;311:20202. doi:10.1001/jama.2013.286141

11 Schmidt ML, Spencer MD, Davidson LE. Patient, Provider, and Practice Characteristics Associated with Inappropriate Antimicrobial Prescribing in Ambulatory Practices. Infect Control Hosp Epidemiol 2018;39:307-15. doi:10.1017/ice.2017.263

12 Klein EY, Van Boeckel TP, Martinez EM, et al. Global increase and geographic convergence in antibiotic consumption between 2000 and 2015. Proc Natl Acad Sci U S A 2018;115:E3463-70. doi:10.1073/pnas.1717295115

13 Hicks LA, Bartoces MG, Roberts RM, et al. US outpatient antibiotic prescribing variation according to geography, patient population, and provider specialty in 2011. Clin Infect Dis 2015;60:1308-16.

14 Arizpe A, Reveles KR, Aitken SL. Regional variation in antibiotic prescribing among medicare part D enrollees, 2013. BMC Infect Dis 2016;16:744. doi:10.1186/s12879-016-2091-0

15 Zhang Y, Steinman MA, Kaplan CM. Geographic variation in outpatient antibiotic prescribing among older adults. Arch Intern Med 2012;172:1465-71. doi:10.1001/archinternmed.2012.3717

16 Lessa FC, Mu Y, Bamberg WM, et al. Burden of Clostridium difficile infection in the United States. N Engl J Med 2015;372:825-34. doi:10.1056/NEJMoa1408913

17 McCaig LF, Hughes JM. Trends in antimicrobial drug prescribing among office-based physicians in the United States. JAMA 1995;273:214-9. doi:10.1001/ jama.1995.03520270048030

18 McCaig LF Besser RE, Hughes IM. Antimicrobial-Drug prescription in ambulatory care settings, United States, 1992-2000. Emerg Infect Dis 2003 [cited 2018 Mar 19];9(4). https://wwwnc.cdc.gov/eid/ article/9/4/02-0268 article

19 Roumie CL, Halasa NB, Grijalva CG, et al. Trends in antibiotic prescribing for adults in the United States--1995 to 2002. J Gen Intern Med 2005;20:697-702. doi:10.1111/j.1525 $1497.2005 .0148 x$

20 Steinman MA, Gonzales R, Linder JA, Landefeld CS. Changing use of antibiotics in community-based outpatient practice, 1991-1999. Ann Intern Med 2003;138:525-33. doi:10.7326/0003-4819-138-7200304010-00008

21 Grijalva CG, Nuorti JP, Griffin MR. Antibiotic prescription rates for acute respiratory tract infections in US ambulatory settings. JAMA 2009:302:758-66. doi:10.1001/jama.2009.1163

22 Suda KJ, Hicks LA, Roberts RM, Hunkler RJ, Matusiak LM, Schumock GT. Antibiotic Expenditures by Medication, Class, and Healthcare Setting in the United States, 2010-2015. Clin Infect Dis 2018;66:185-90. doi:10.1093/cid/cix773

23 Van Boeckel TP, Gandra S, Ashok A, et al. Global antibiotic consumption 2000 to 2010: an analysis of national pharmaceutical sales data. Lancet Infect Dis 2014;14:742-50. doi:10.1016/S1473 3099(14)70780-7
24 Blue Cross Blue Shield. Antibiotic prescription fill rates declining in the US. 2017 [cited 2018 March 2019]. https://www.bcbs.com/thehealth-of-america/reports/antibiotic-prescription-rates-declining-inthe-US

25 Durkin MJ, Jafarzadeh SR, Hsueh K, et al. Outpatient antibiotic prescription trends in the United States: A national cohort study. Infect Control Hosp Epidemiol 2018;39:584-9. doi:10.1017/ ice. 2018.26

26 Lee GC, Reveles KR, Attridge RT, et al. Outpatient antibiotic prescribing in the United States: 2000 to 2010. BMC Med 2014;12:96. doi:10.1186/1741-7015-12-96

27 Mues KE, Liede A, Liu J, et al. Use of the Medicare database in epidemiologic and health services research: a valuable source of real-world evidence on the older and disabled populations in the US. Clin Epidemiol 2017;9:267-77. doi:10.2147/CLEP.S105613

28 Centers for Disease Control and Prevention. Ambulatory health care data. [cited 2018 March 2019]. https://www.cdc.gov/nchs/ahcd/ index.htm

29 MacKinnon JG, White H. Some heteroskedasticityconsistent covariance matrix estimators with improved finite sample properties. J Econom 1985;29:305-25. doi:10.1016/03044076(85)90158-7

30 Mandell LA, Wunderink RG, et al, Anzueto Alnfectious Diseases Society of AmericaAmerican Thoracic Society. Infectious Diseases Society of America/American Thoracic Society consensus guidelines on the management of community-acquired pneumonia in adults. Clin Infect Dis 2007;44(Suppl 2):S27-72. doi:10.1086/511159

31 Lynch JPIII, Martinez FJ. Clinical relevance of macrolide-resistant Streptococcus pneumoniae for community-acquired pneumonia. Clin Infect Dis 2002;34(Suppl 1):S27-46. doi:10.1086/324527

32 Ray WA, Murray KT, Hall K, Arbogast PG, Stein CM. Azithromycin and the risk of cardiovascular death. N Engl J Med 2012;366:1881-90. doi:10.1056/NEJMoa1003833

33 Burke JP. Antibiotic resistance--squeezing the balloon? JAMA 1998;280:1270-1. doi:10.1001/jama.280.14.1270

34 Seppälä H, Klaukka T, Vuopio-Varkila J, et al, Finnish Study Group for Antimicrobial Resistance. The effect of changes in the consumption of macrolide antibiotics on erythromycin resistance in group $A$ streptococci in Finland. N Engl J Med 1997;337:441-6. doi:10.1056/ NEJM199708143370701

35 US Food \& Drug Administration. FDA Drug Safety Communication: FDA updates warnings for oral and injectable fluoroquinolone antibiotics due to disabling side effects. 2016. https://www.fda.gov/ Drugs/DrugSafety/ucm511530.htm

36 Riley GF, Levy JM, Montgomery MA. Adverse selection in the Medicare prescription drug program. Health Aff (Millwood) 2009;28:1826-37. doi:10.1377/hlthaff.28.6.1826

37 Riedle BN, Polgreen LA, Cavanaugh JE, Schroeder MC, Polgreen PM. Phantom prescribing: examining the frequency of antimicrobial prescriptions without a patient visit. Infect Control Hosp Epidemiol 2017;38:273-80. doi:10.1017/ice.2016.269

38 Dolk FCK, Pouwels KB, Smith DRM, Robotham JV, Smieszek T. Antibiotics in primary care in England: which antibiotics are prescribed and for which conditions? J Antimicrob Chemother 2018;73(suppl_2):ii2-10. doi:10.1093/jac/dkx504

39 Department of Health and Human Services. Coding trends of Medicare evaluation and management services. 2012. Report No: OEl-04-10-00180.

40 Pichichero ME. Dynamics of antibiotic prescribing for children. IAMA 2002-287:3133-5. doi:10.1001/jama.287.23.3133

41 Price L, Gozdzielewska L, Young M, et al. Effectiveness of interventions to improve the public's antimicrobial resistance awareness and behaviours associated with prudent use of antimicrobials: a systematic review. J Antimicrob Chemother 2018;73:1464-78. doi:10.1093/jac/dky076

Web appendix: Supplementary materials 